\title{
Sprichwörtliche Weisheiten
}

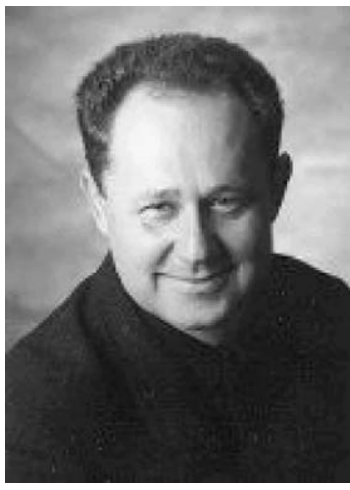

Prof. Dr. med. Manfred Wildner

Biblipografie

DOI http://dx.doi.org/ 10.1055/s-0032-1331174 Gesundheitswesen 2012; 74: 689-690

(c) Georg Thieme Verlag KG Stuttgart · New York

ISSN 0941-3790

\section{Korrespondenzadresse}

Prof. Dr. med. Manfred Wildner

Bayerisches Landesamt für Gesundheit und Lebensmittelsicherheit

Veterinärstraße 2

85762 Oberschleißheim

manfred.wildner@lgl.bayern.de
„Spare in der Zeit, dann hast du in der Not!“ dieses und ähnliche Sprichwörter geben in der Parömiologie (Sprichwörterkunde) eine Lebensregel oder allgemeine Weisheit mithilfe eines kurzen Satzes wider. Wobei sich die Frage stellt, wie sich diese Weisheit richtig anwenden lässt: Wie gehen wir z.B. mit widersprüchlichen Spruchweisheiten um, wie „Gegensätze ziehen sich an“, in Konkurrenz mit "Gleich und Gleich gesellt sich gern"? Manches Sprichwort würden wir auch heute eher als Vorurteil denn als Klugheitsregel bezeichnen, z.B. „was der Mann mit dem Wagen einfährt, trägt die Frau mit der Schürze hinaus". Oder "Jung gefreit hat niemand gereut" wohl eher eine „tollkühne Behauptung“ (Wolf Schneider), die einer empirischen Überprüfung schwerlich standhalten dürfte. In Abgrenzung zum Zitat oder zum geflügelten Wort kommt dem Kriterium der „wiederholten Rede“ große Bedeutung zu.

Solche „wiederholte Reden“ gibt es auch im Gesundheitswesen. Eine dieser sprichwörtlichen Weisheiten ist die wiederholte Feststellung, Prävention spare Ausgaben im Gesundheitswesen. Eine Aussage, die plausibel klingt, unter Aspekten einer salutogenetischen Neuausrichtung im Gesundheitswesen ein hochwillkommenes Argument darstellt und auch gut dazu passt, dass Gesundheit ohnehin besser ist als Krankheit.

Doch wie steht es mit der empirischen Basis dieser Spar-These? Sie steht im Kontext von Fragen der Priorisierung von Leistungen im Gesundheitswesen, bei der nicht nur ökonomische, sondern auch ethische, politische und pragmatische Aspekte eine wichtige Rolle spielen. Doch sehen wir uns die angesprochene ökonomische Seite der Sache an. Bei einer ökonomischen Evaluation neuer Handlungsoptionen im Gesundheitswesen sind 3 Hauptfragen die nach der Wirksamkeit (ist das Neue besser oder zumindest gleich gut, auch unter Berücksichtigung der Nebenwirkungen?), die nach der Wirtschaftlichkeit (werden kostengünstig Ausgaben eingespart oder sind evtl. Mehrkosten zumindest durch einen Gewinn an Gesundheit gerechtfertigt?) und die Frage nach den ökonomischen Gewinnern und Verlierern (wer trägt die Kosten, wer hat den Nutzen?). Bisweilen gelingt es, im Einklang mit der ökonomischen Theorie, externalisierte (Gesundheits-) Kosten wieder zurückzuführen und die präventiven Mehrkosten beim Verursacher anzusiedeln, wie dies mit Sicherheitsgurten und Russfiltern im Straßenverkehr gelungen ist. Aus Sicht der gesamten Produktionskosten „Kleingeld“ im Dienst der Gesundheit („small change for health“), aus
Sicht der gesundheitlichen Outcomes ein großer Erfolg („make healthy choices easy choices“). Verschiedene Ansätze und Begrifflichkeiten sind in der Prävention zu unterscheiden: Primär-, Sekundär- und Tertiärprävention oder auch Primordialprävention und Krankheitsfrüherkennung, Verhaltens- und Verhältnisprävention, universelle, selektive und indizierte Prävention. Bei einer Beschränkung auf die Primärprävention, also die Verhinderung von neuen Krankheitsfällen, bietet eine Unterscheidung nach kontextbezogenen gegenüber personenzentrierten Interventionsmaßnahmen und bei letzteren eine Unterscheidung zwischen klinischen und nichtklinischen („nicht medizinischen“) Maßnahmen interessante Erkenntnisse [1]. Umwelt- bzw. kontextorientierte Verhältnisprävention scheint in einem hohen Prozentsatz kostengünstig, d.h. gegenüber dem Status quo mit Einsparungen verbunden zu sein. Hier trifft das oben zitierte sprichwörtliche Wissen nach Studienlage zumindest in der Hälfte der Fälle aus gesamtgesellschaftlicher Sicht zu. Ob auch Einsparungen für das Gesundheitswesen realisiert werden können, ist eine eigene Frage. Bei verhältnisorientierten Maßnahmen sind die Investitionen teilweise einmaliger Natur, regulatorische Maßnahmen sind häufig mit vergleichsweise niedrigen, wenn auch wiederkehrenden Kosten verbunden. Beispiele dafür sind eine umfassende Nichtraucherschutzgesetzgebung, die Regulierung der Abgabe von alkoholischen Getränken oder der Bann von Transfettsäuren. Gut belegt sind auch die positiven Effekte einer hohen Besteuerung von Tabak, die Förderung von Freizeitsport und zusätzliche Zeit für Sportunterricht an Schulen [2].

Bisher noch wenig unter der Rubrik „Verhältnisprävention" diskutiert sind Maßnahmen im Gesundheitswesen selbst: Die Prävention von Über-, Unter- und Fehlversorgung, wie z.B. durch Zweitmeinungen vor Operationen, durch Angebote zur partizipativen Entscheidungsfindung sowie durch Analysen qualitätsabträglicher Variationen der Versorgungsrealität im Rahmen einer aktiven Versorgungsforschung mit Feed-back Elementen. Vielversprechend sind auch Maßnahmen zur Vermeidung nosokomialer Infektionen, zur Patientensicherheit in operativen Fachgebieten und zum allgemeinen Risikomanagement in Einrichtungen der gesundheitlichen Versorgung. Bei personenzentrierten Ansätzen finden sich dem gegenüber Einsparungen nur für eine von etwa 7 untersuchten Maßnahmen. Als prioritär für den Bereich der personenzentrierten klinischen Prävention werden Aspirin bei Hoch-Risi- 
kopersonen, Impfprogramme bei Kindern, Beratungen zur Tabakabstinenz, Screening auf Darmkrebs bei Personen über 50 Jahre, Pneumokokken-Impfungen bei Personen ab 65 Jahren und ein Chlamydien-Screening bei jungen Frauen eingestuft [3]. Im Allgemeinen werden im Bereich der personenzentrierten Prävention Lebensjahre und Lebensqualität mit zusätzlichen Ausgaben erkauft, wobei eine Unterversorgung mit grundsätzlich anerkannten Präventionsmaßnahmen zu konstatieren ist $[4,5]$. Verhältnisorientierte Maßnahmen und personenzentrierte Maßnahmen ergänzen sich dabei gegenseitig. Eine Analyse des Rückgangs der kardiovaskulären Mortalität seit Mitte der zweiten Hälfte des 20. Jahrhunderts kommt zu dem Ergebnis, dass etwa die Hälfte bis zwei Drittel davon verhältnisorientierten Maßnahmen zugerechnet werden kann, ein Drittel bis die Hälfte einer Verbesserung der klinischen Behandlungsoptionen [6].

Die Anwendung der sprichwörtlichen Weisheiten im Kontext von Prävention und Gesundheitsförderung erfordert also Unterscheidungsvermögen. Fraglich ist, ob ein freier Markt alleine in der Lage ist, das dafür erforderliche weitsichtige Handeln zu erzeugen. Unser Gesundheitssystem ist ein „System komplexer Vielfachsteuerung“ (Alber), mit „problembezogenen und bereichspezifischen Mischsteuerungen“ (Rosenbrock). Diese erfordern geduldiges und beständiges Adjustieren im Rahmen der Steuerungspluralität, nicht ungeduldiges Revolutionieren. Miteinander konkurrierende Werte stehen dabei im Wettbewerb: Ein Such- und Lernprozess auch mit dem Ziel des Ausgleichs als sozialethischer Bestrebung. Investitionsüberlegungen, die auf das Hier und Jetzt und bei Zukunftsorientierung auf den eigenen Nutzen ausgerichtet sind (,hic et nunc et ego“), stehen in fruchtbarer Konkurrenz mit einer Wohlfahrtsorientierung in Richtung auf benachteiligte Populationen, mittel- und langfristige Zeithorizonte und altruistische Nutzenorientierungen (,ibi et post et alter").

Ein Sparen um jeden Preis mag in der Prävention ohnehin nicht die beste Zielsetzung sein. Gesundheitsressourcen lassen sich nicht nur als Solidarbeiträge oder Gaben der Barmherzigkeit verstehen. Sie sind auch Talente im buchstäblichen wie im übertragenen Sinn, welche uns treuhändisch anvertraut sind und nicht vergraben werden sollten. Finanzielle Ressourcen, Talente im ursprünglichen Verständnis des biblischen Gleichnisses (Matthäus 25, 14-30), können klug in gesundheitsrelevante Kompetenzen und Kapazitäten überführt werden, Talente im übertragenen Sinn (siehe auch [7]). Diese Investitionen in das Gemeinwohl tragen bei kluger Allokation vielfache Früchte, wobei vielfache Produktionswege für Gesundheit zu berücksichtigen sind. Vielleicht sollte das eingangs zitierte Sprichwort „Spare in der Zeit, dann hast du in der Not! " im Kontext von Gesundheitsschutz, Prävention und Gesundheitsförderung ergänzt werden durch einen Verweis auf diesen Matthäus-Effekt: „Denn wer da hat, dem wird gegeben".

Mit aller Hochachtung vor der angesprochenen Volksgruppe sei auch die Geschichte des schwäbischen Bergwanderers erzählt, welcher bei einer Gletscherüberquerung in eine Spalte fiel. Den Zuruf des Rettungstrupps, welcher sich mit einem „Hier ist die Bergwacht! “ bei ihm meldete, beantwortete er mit einem knappen „Mir gäbe nix!“ Offenkundig eine begrenzte, auf unangemessene Sparsamkeit hin orientierte Rationalität: hier hätte sich eine Gesundheitsausgabe durch deutlich verbesserte Einkommensaussichten in der Zukunft sehr wohl gelohnt.

Unstrittig ist, dass uns Erfolge im Bereich des Gesundheitsschutzes, der Prävention und Gesundheitsförderung vor neue Herausforderungen stellen, wie dies durch die effektive Kontrolle vieler
Infektionskrankheiten die Zunahmen chronischer Krankheiten gezeigt haben. Doch ist ein damit gefordertes füreinander Dasein, über Generationen hinweg, nicht ein Grundmuster sozialer Gruppen, wie z.B. in den Familien als Keimzellen des Staates? Auch hier findet sich ein personen- und generationenübergreifendes Miteinander: der traditionstragenden älteren Generation als storytellers (Geschichtenerzähler), der berufstätigen ElternGeneration als breadwinners (Ernährer) und der Kinder als investitionsbedürftige „Start-Up Unternehmungen“.

So mag die Antwort auf die eingangs gestellte Frage, ob sich durch Prävention Gesundheitsausgaben sparen lassen, lauten: unmittelbare Einsparungen durch Präventionsausgaben sind nur zum Teil möglich. Wahrscheinlich ist jedoch, dass durch evidenzbasierte Prävention in einer gesamtgesellschaftlichen Betrachtung zusätzlicher Nutzen und zusätzliche Ressourcen durch eine erhöhte Produktivität generiert werden können, welche dann in Teilen auch dem Gesundheitswesen zur Verfügung stehen und zu einem positiven Saldo führen können.

Derartig rationalem Handeln dienen die Beiträge in dieser Ausgabe unserer Zeitschrift, unter Rückgriff auf den wissenschaftlichen Diskurs: Zu Schulformen und sozialem Kapital in der Adoleszenz, zur Bedeutung kleinräumiger Lebensverhältnisse, zu gesundheitlichen Belastung durch Lärm, zu Einstellungen gegenüber der HPV-Impfung und Früherkennungsangeboten von Brust- und Gebärmutterhalskrebs, zu psychosozialen Krebsberatungsstellen, zu krebskranken Eltern und ihren minderjährigen Kindern, zum Referenznetzwerk für invasive bakterielle Infektionen und zum Transfer von Forschungsergebnissen in die medizinische Praxis. Hinzu kommt eine unter Beteiligung verschiedener Fachgesellschaften abgestimmte Stellungnahme zu Public Health in Deutschland.

Um noch einmal den Bogen zum Anfang zu schlagen: die sprichwörtliche Weisheit, dass womöglich Frauen das mit der Schürze hinaustragen, was der Mann mit dem Wagen einfährt, ist nicht nur unter Gender-Aspekten problematisch, sondern auch in der Sache womöglich sehr verkürzt. Sozialwissenschaftliche Untersuchungen, insbesondere auch in Entwicklungsländern, haben gezeigt, dass Frauen zur Verfügung gestellte Gelder in der Regel wirtschaftlicher im Sinne kluger Investition ausgeben als Männer. Insofern wäre also zu prüfen, ob dass, was in der Schürze herausgetragen wurde, nicht womöglich kluge Investition in Bildung und Gesundheitsförderung und damit in die Steigerung der allgemeinen Wohlfahrt ist: „Investiere in der Zeit, dann hast Du in der Not!“.

\section{Literatur}

1 Chokshi D, Farlay TA. The Cost-Effectivness of Environmental Approaches to Disease Prevention. N Eng J Med 2012; 367: 295-297

2 Brownson RC, Haire-Joshu D, Luke DA. Shaping the context of health: a review of environmental and policy approaches in the prevention of chronic diseases. Annu Rev Public Health 2006; 27: 341-370

3 Maciosek MV, Coffield $A B$, Edwards NM et al. Priorities among effective Clinical Preventive Services: results of a systematic review and analysis. Am J Prev Med 2006; 31: 52-60

4 Russell BL. Prevention's Potential for slowing the growth of Medical Spending. National Coalition of Health Care 2007;URL www.nchc.org

5 Cohen JT, Neumann PJ, Weinstein MC. Does Preventive Care save money? Health economics and the presidential candidates. NEJM 2008; 358: 661-663

6 Ford ES, Capewell S. Proportion of the Decline in Cardiovascular Mortality Disease due to Prevention versus Treatment: Public Health versus Clinical Care. Annu Rev Public Health 2011; 32: 5-22

7 Kuhn J. Taten ohne Daten? Ein Kommentar zur Rolle der Gesundheitsberichterstattung in der Prävention. Jahrbuch für Kritische Medizin 2006; 43: 25-39 Abrufbar unter http://www.josephkuhn.de/pdf/Ta ten_ohne_Daten.pdf 